\title{
Vasomotor response in human subjects: Conditioning and pseudo-conditioning'
}

\begin{abstract}
Abstraet
The vasomotor responses to a light of 9 Ss administered a series of light-sound pairings did not differ in the course of over 40 trials from those of 6 Ss administered the same stimuli unpaired. Classical conditioning did not occur; the occurrence of (unpaired) conditioned and unconditioned stimuli was sufficient to explain the behavior.
\end{abstract}

\section{Problem}

This paper reports an unsuccessful attempt to demonstrate that vasomotor conditioning is possible with both pseudo-conditioning and breathing controlled.

The effect of pseudo-conditioning (Grether, 1938) should be evaluated in classical conditioning experiments to establish that past temporal pairings of the conditioned stimulus (CS) with the unconditioned stimulus (UCS) were necessary to establish the CS as an eliciting stimulus. One appropriate technique is to compare responses produced by the standard conditioning procedure with those produced by administering CS and UCS unpaired, in random order, separated by the usual inter-trial interval.

A second class of artifacts is also important in attempts to condition vasomotor responses (VMRs). Some changes in breathing produce VMRs. What appears to be a conditioned VMR, an autonomic response, may instead be a by-product of a conditioned change in rate or depth of respiration, a response of the skeletal muscles. Considerable control of human respiration can be achieved by instruction, e.g., by requiring Ss to breathe in rhythm with a metronome.

Previous studies of vasomotor conditioning, e.g., Menzies (1937), Shmavonian (1959), and Fromer (1963), have failed to control for one or both of these artifacts, and so have failed to demonstrate unambiguously that vasomotor conditioning is possible. Shmavonian (1959) used shock for UCS and a delay conditioning procedure. He gave human Ss 10 trials of CS-UCS pairings, followed by 10 trials of CS alone, and reported rapid conditioning and gradual and incomplete extinction. He reported that respiration rate was not correlated with conditioning. However, he did not control for pseudoconditioning. Fromer (1963), using rabbits, controlled for pseudo-conditioning but not for possible conditioned changes in breathing. He may have conditioned some form of brief respiratory arrest to the CS, with concomitant vasoconstriction in the rabbits' ears.

\section{Method}

The three experimental groups in the present experiment were:

Group I: Conditioning. The CS was presented for 17 sec., the last $2 \mathrm{sec}$. of which were simultaneous with the UCS. Nine Ss run under this procedure received from 40 to 113 pairings.

Group II: Pseudo-conditioning. Either the CS was presented for $17 \mathrm{sec}$, or the UCS for $2 \mathrm{sec}$. The order of presentation of the stimuli was random. Six Ss run under this procedure received from 45 to $73 \mathrm{CSs}$.

Group III: Pseudo-conditioning, 10-trial group. For this group, the procedure paralleled that in Shmavonian's study. The eight Ss in this group saw $10 \mathrm{CSs}$ under the pseudo-conditioning procedure, followed by 10 successive CSs without any UCSs ("extinction"').

The CS was a $71 / 2-w$ frosted light bulb, fastened in front of $S$ at his eyelevel. The UCS was a $95 \mathrm{db}$ buzz of 2 sec. duration, presented through earphones. The UCS was extremely unpleasant. Three Ss refused to complete the experiment, saying that the stimulus was too noxious.

In the first session, CSs were repeated until $\mathrm{S}$ did not give a VMR to three successive CSs. The third of these negative trials was the first experimental trial. Any $S$ that did not adapt to the CS within the first 20 presentations or that did not give unconditioned VMRs to at least $90 \%$ of the UCSs in any session was discarded. Eleven were discarded for the former reason, and three for the latter. Twenty-three male high school students, 13 to 18 years old, completed the experiment. They were paid for their participation. For all Ss, the inter-trial interval varied from 2 to $3 \mathrm{~min}$. Sessions lasted an hour, with a brief rest period after $30 \mathrm{~min}$.

The Ss were instructed to breath in rhythm with a metronome, which was set at 31 beats per min., providing $151 / 2$ respiratory cycles per min. Depth of respiration was uncontrolled.

A photocrystal plethysmograph similar to Shmavonian's (1959) detected the change in the volume of blood flowing through the tip of S's index finger. The S's heart rate and breathing rate were also monitored. Further details concerning the apparatus and procedure are available in Stolz (1963).

All VMRs following CS onset and prior to UCS offset with latency at least equal to the latency of the unconditioned VMR (3 to $4 \mathrm{sec}$.) were scored as responses to the CS, providing that they satisfied two other criteria: (a) base to peak height greater than the height of the change in volume of blood due to the pulse which preceded stimulus onset, and (b) duration of at least $10 \mathrm{sec}$.

\section{Results}

Figure 1 shows the percentage of Ss giving a VMR on each trial from 2 to 40 . Clearly the performance of Ss in the conditioning group did not differ from that of those in the pseudo-conditioning group. 
(It was not always possible to determine whether an $\mathrm{S}$ had responded on a particular trial, so the total $\mathrm{N}$ for some trials is less than that specified in the figure captions. The initial trial is omitted because the conditioning series always began on a trial in which $\mathrm{S}$ gave no response to the $\mathrm{CS}$.)

Six Ss in Group I received from 24 to 73 trials beyond the 40 reported in Fig. 1 . They continued to respond to the CS as they had during the period covered by Fig. 1 .

Following the first 15 trials, Ss' tendencies to respond to the CS stabilized. Only one $S$ showed an increased frequency of response as conditioning trials progressed. When shifted to pseudo-conditioning conditions, he responded less frequently than when stimuli were paired (see Stolz, 1963).

The Ss administered a procedure paralleling that of Shmavonian (Group III) produced results like Shmavonian's. In Fig. 2, data from the present experiment are presented.

\section{Diseussion}

This experiment failed to demonstrate classical conditioning of the VMR in human Ss whose breathing was regulated. No difference was observed between the behavior of the conditioning and the pseudo-conditioning groups. Hence, explanation of the observed behavior need refer only to the occurrence of (unpaired) CSs and UCSs, not to the pairing of the stimuli.

The similarity of Shmavonian's results to those of this experiment suggests that the temporal relationship between the CS and the UCS played no role in his results either. Shmavonian might well have obtained the same results with unpaired stimuli.

The conditions of the present experiment were favorable for vasomotor conditioning. All Ss gave unconditioned VMRs to the UCS on at least $90 \%$ of trials. For some Ss, an unconditioned VMR occurred immediately following a CS on 40 to 113 trials. Nevertheless, only one $\mathrm{S}$ out of nine gave any indication that the pairing of

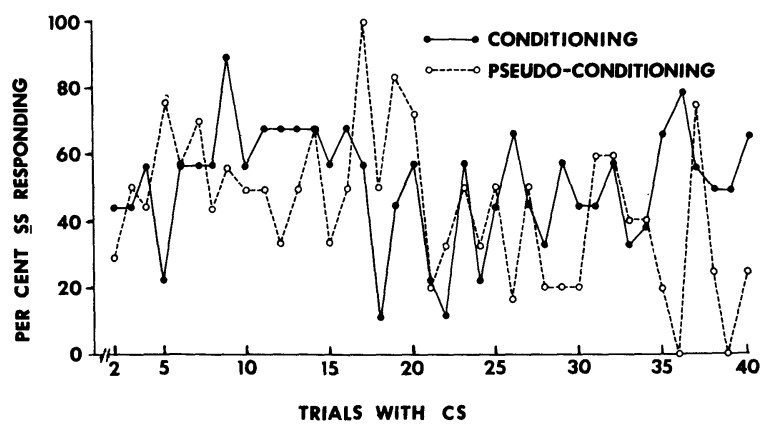

Fig. 1. Percentage of Ss giving vasomotor responses on the first 40 trials in the conditioning and pseudo-conditioning groups. For conditioning trials, $\mathrm{N}=9$; for pseudo-conditioning trials, $\mathrm{N}=14$ on trials 2 to $10, \mathrm{~N}=6$ on trials 11 to 40 .

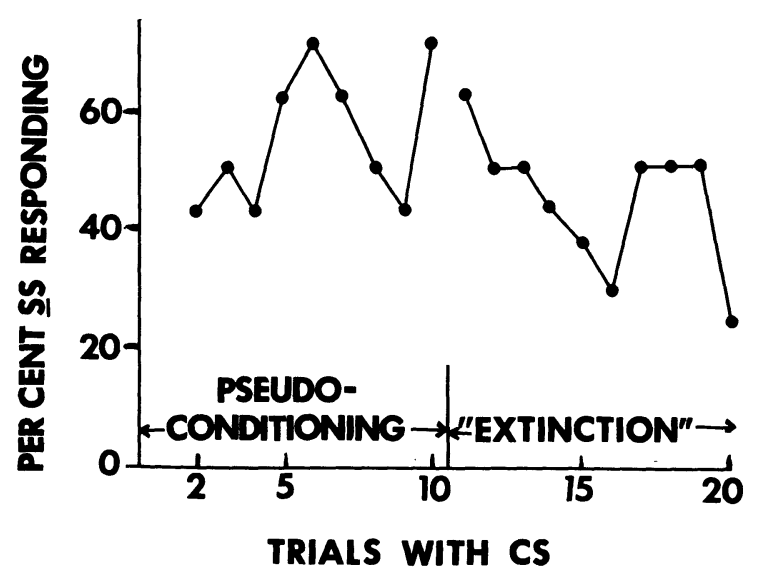

Fig. 2. Percentage of Ss responding to CS on pseudo-conditioning and "extinction" trials, Group III, $\mathrm{N}=8$.

CS with UCS influenced his behavior, and for him the evidence is weak.

Further controlled studies need to be made of vasomotor conditioning, using more trials, other stimuli, and other inter-stimulus intervals. However, the present results suggest that previous experiments purporting to demonstrate vasomotor conditioning should be reexamined, for the earlier results may be artifactual, produced by failure to control for breathing, pseudoconditioning, or both.

References

FROMER, R. Conditioned vasomotor responses in the rabbit. J. comp. physiol. Psychol., 1963, 56, 1050-1055.

GRETHER, W. F. Pseudo-conditioning without paired stimulation encountered in attempted backward conditioning. J. comp. Psychol., 1938, 25, 91-96.

MENZIES, R. Conditioned vasomotor responses in human subjects. J. Psychol., 1937, 4, 75-120.

SHMAVONIAN, B. M. Methodological study of vasomotor conditioning in human subjects. J. comp. physiol. Psychol., 1959, 52, 315-321. STOLZ, S. B. Conditioning and pseudo-conditioning of the vasomotor response in human subjects. Unpublished doctoral dissertation, University of Washington, 1963.

Note

1. This report is based upon a Ph.D. dissertation submitted to the Department of Psychology of the University of Washington, Seattle. I am grateful to my sponsor, R. B. Loucks, for his help and encouragement during the course of the study. I also thank D. M. Baer for his advice and criticism, and Ward Edwards for his critical reading of the manuscript. This investigation was supported in part by a Public Health Service fellowship (MPM-16,233) from the National Institutes of Health, Public Health Service. 